\title{
Crack propagation along polymer/non-polymer interfaces
}

\author{
Willem-Pier Vellinga · Alexander Fedorov • \\ Jeff T. De Hosson
}

Received: 3 April 2007 / Accepted: 24 April 2007 / Published online: 7 March 2008

(C) The Author(s) 2008

\begin{abstract}
Mechanisms of the propagation of crack fronts along interfaces between a glassy polymer and metal or glass are discussed. Specifically, the systems studied are Poly-Ethylene Terephthalate (PETG) spin-coated on Al, PETG-glass and PETG hot-pressed on Cr-sputtered glass. Cracks studied propagate in an Assymetric Double Cantilever Beam (ADCB) geometry. Dependence of microscopic crack front geometry on propagation speed is found. The local stress state is found to have an impact on macroscopic as well as microscopic crack front geometry. Simple lattice model calculations of propagating crack fronts illustrate the impact of disorder and residual stress state on propagation mechanisms and macroscopic crack front shape respectively.
\end{abstract}

Keywords Adhesion - Crack propagation - Residual stress - ADCB · Interface · Polymer-metal

\section{Introduction}

Novel products that rely on the combination of polymers with other materials such as metals and oxides appear at increasing pace. Examples can be found in microelectronics, in environmental, biochemical and biomedical sensors or in flexible displays and solar cells. More traditional industries are also impacted. Polymer-coated metal sheets for example, increasingly act as a substitute for lacquered tinplates: in packaging of food or aerosols, as car body parts or as coatings in roof and wall cladding. The yield and reliability of these new products are significantly influenced by the interfacial adhesion and the resistance of the interface to delamination (e.g. [1]), and for successful development advances are needed in the control and measurement of interfacial adhesion. The apparently "low-tech" applications such as in food packaging or the automotive industries may present some of the toughest challenges because of the complications arising in the forming stages. This process usually leads to high

W.-P. Vellinga $(\varangle) \cdot$ A. Fedorov · J. T. De Hosson

Department of Applied Physics, Netherlands Institute for Metals Research and Zernike Institute for Advanced Materials, University of Groningen, Nijenborgh 4, Groningen, AG 9747, The Netherlands e-mail: w.p.vellinga@rug.nl 
pressures and high strains and deformation rates that affect the mechanical properties of the polymer during forming.

Since the product and production route are coupled intimately techniques are needed to measure adhesion on the resulting product as well as on the feedstock of sheet. During the forming step the metal surface (typically of a steel or aluminum alloy) may roughen appreciably due to plastic deformation (see e.g.[2]) inducing local stress concentrations and deformation in the polymer near the interface [3]. Local delamination techniques such as described in [4] may present a way forward in establishing the effects of such treatments on the adhesion.

Measurements in realistic geometries are also important because elastic energy released during delamination (for example due to thermal or shrinkage stresses) may have been stored far away from the crack tip causing the release rate to effectively depend on macroscopic sample properties and dimensions. The elastic energy released is dissipated in the delamination process on several scales. For the practical adhesion of a polymer on a metal the character of bonding at the interface itself is not the only relevant issue. The length of the polymer chain physically interacting (i.e. the degree of entanglement) with the polymer coating and the mechanical properties of the polymer coating itself together determine the maximum loads transferred to the interface, and the mechanisms that dissipate energy (e.g. [5]). For a modeling approach the coupling of these scales requires reconciliation of views of crack propagation based on continuum mechanics with statistical physics so as to include the effects of disorder. Disorder may be due to local fluctuations in geometry, (e.g. layer thickness, roughness), material properties, or interface chemistry and show up as fluctuations in the local energy release rate associated with the interface crack. The degree of disorder, local stress concentrations near irregularities on the front, elastic interaction (potentially of long range character) along the front are all expected to influence the crack shape and crack dynamics (for a review see e.g. [6]). Experiments imaging cracks propagating along weak PS-PS interfaces have revealed scaling behavior of crack front shape and crack front dynamics [7, 8]. Also it has been hypothesized that differences in the strength of disorder may impact crack propagation mechanisms [9]. Such detailed exploration of crack propagation mechanisms seems especially relevant for lifetime prediction where quantities accessible from continuum mechanics (stresses, stress intensity factors, energy release rates) may be related to the interplay between disorder and stress-aided thermally activated processes [10].

In this paper experimental results illustrate a number of issues touched upon above: the impact of residual stress state on crack propagation (apparent both on a macroscopic and microscopic level), the impact of disorder on crack propagation. Simple lattice model calculations are used to qualitatively interpret some of the results.

\section{Experiment}

Crack propagation is performed in an Asymmetric Double Cantilever Beam (ADCB) set-up mounted in a reflection optical microscope. For details on ADCB experiments and related issues reference is made to [11] and [12]. In ADCB the energy release rate $G(a)$ follows from a measurement of the crack length $a$ and use of [12] :

$$
G(a)=\frac{\left(3 \Delta^{2} E_{1} E_{2} h_{1}^{3} h_{2}^{3}\right)\left(C_{2}^{2} E_{1} h_{1}^{3}+C_{1}^{2} E_{2} h_{2}^{3}\right)}{8 a^{4} \Lambda^{2}}
$$


with $E_{i}$ Young's moduli, $\Delta$ the fixed crack opening set by the thickness of a razor blade, $\Lambda=C_{1}^{3} E_{2} h_{2}^{3}+C_{2}^{3} E_{1} h_{1}^{3}$ and $C_{i}=1+0.64 \frac{h_{i}}{a}$ and $\mathrm{i}=1,2$. In practice $a \gg h_{1}, h_{2}$. Also at all times, for the remaining adhered portion of the beam $L, L \gg a$ should hold.

Several types of samples were used. One type consists of glassy Poly-Ethylene Terephthalate (PETG) with a thickness of $15 \mu \mathrm{m}$, spin-coated on Cr-coated steel, another of PETG spin-coated on $\mathrm{Al}$ with a thickness of $15 \mu \mathrm{m}$. After spin-coating these two types of samples are dried at $80^{\circ} \mathrm{C}$ for a few hours, and subsequently pressure bonded to a $1 \mathrm{~mm}$ thick glass support (for $240 \mathrm{~s}$, at $140^{\circ} \mathrm{C}$ and $1.5 \mathrm{MPa}$ ). Typical substrate dimensions for all types of samples are $10 * 50 * 2 \mathrm{~mm}$. In these types of samples a crack may propagate along the PET-glass interface as well as along the rough and anisotropic (due to ridges in the rolling direction) PET-metal interface ( $\mathrm{rms} \sim 1 \mu \mathrm{m}$ ). In a third type of sample cracks were forced to run along a smooth interface, between $\mathrm{Cr}$ (possibly oxidized) and a thick ( $300 \mu \mathrm{m})$ PETG layer. The Cr layer was deposited on a glass slide in a TEER unbalanced magnetron PVD apparatus. The glass was initially cleaned with soap, then with chloroform and eventually etched in a $2 \% \mathrm{HF}$ solution. It was glued with silver paste to a sample holder and transferred to the PVD vacuum. A cleaning step with high energy Ar ions was performed after which the $\mathrm{Cr}$ layer was deposited. The PETG film (Vivak, Bayer) was applied at $150^{\circ} \mathrm{C}$ under a pressure of about $0.5 \mathrm{MPa}$ for $300 \mathrm{~s}$. Pressure was exerted on the PETG via a glass slide covered with a spincoated layer of methyl nonafluoro(iso)butyl ether to prevent sticking to the PETG. Subsequently the sample was left to cool on a metal block. The glass substrate was cut from the bottom producing a crack running to the PETG layer. Bending the two glass pieces away from each other produced a precrack at the interface. Subsequently the PETG layer was cut from the top releasing two samples. A razor blade (thickness $100 \mu \mathrm{m}$ ) was inserted at the interface.

The crack front is observed through the PETG (and glass cover if present) with a CCD camera $(1376 * 1032$ pixels, $3 * 8$ bit, rate $1 \mathrm{~Hz})$. In the experiment the position of the knife is fixed, and the sample is clamped in one of the clamps of the tensile stage. The movement of the clamps may not be entirely smooth nor be aligned perfectly with the coordinate system of the image. Standard image correlation techniques allow determination of any movement of the sample between two images, and so the movement of the front with respect to the sample during the same time can be determined [9].

\section{Simulations}

A lattice model [13] is used to qualitatively interpret the experimental results. It uses a simple elastic material model but nevertheless captures the following important aspects relevant to crack propagation in delamination: disorder, stress redistribution along the front, effect of thickness and bending of the coating. The model consists of three layers: substrate, interface and coating. Elements of two different types make up the model. The coating and substrate are built of simple linear elastic springs that are not allowed to break. For the coating a 3D network provides bending stiffness, which captures thickness effects in delamination The substrate consists simply of a single layer of springs restricted to a plane of constant y (see Fig. 1).

The second type of elements is used at the interface. These elements are linear elastic leaf-springs that can also transfer loads perpendicular to their axis. Elements in the interface are considered to break at a predetermined critical elongation derived from an axial breaking force, $\mathrm{F}_{b n}$, defined as: 


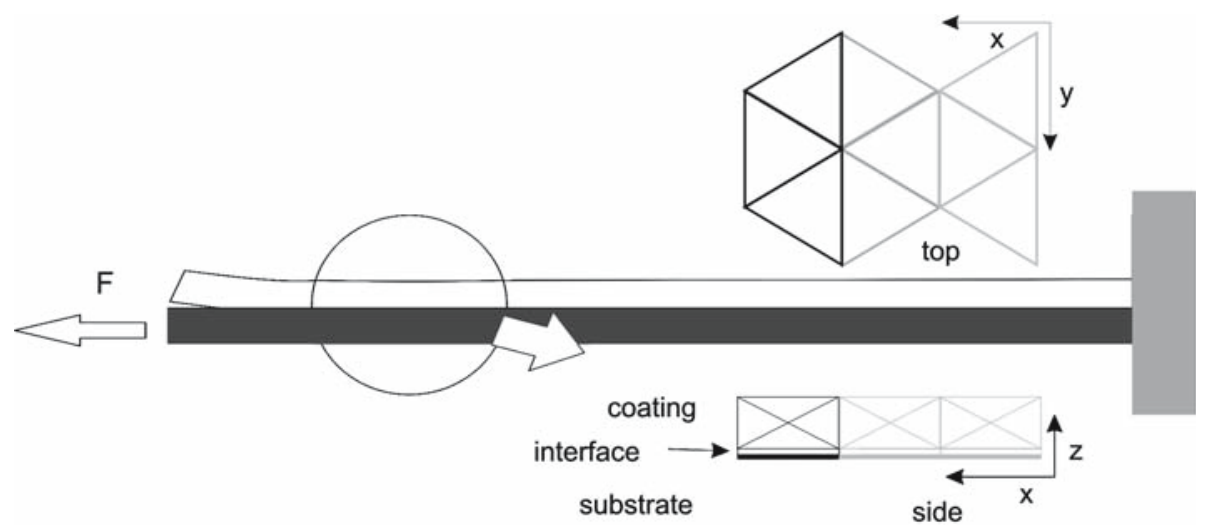

Fig. 1 Sketch of the lattice model used. To propagate a crack along the interface a force $\mathrm{F}$ is applied to the substrate in the $\mathrm{x}$ direction. Periodic boundary conditions can be applied along $\mathrm{y}$. Biaxial stresses can be simulated superimposing forces on the substrate along $\mathrm{x}$ and $\mathrm{y}$

$$
\mathrm{F}_{b, n}=\mathrm{F}_{b}+\mathrm{W} \mathrm{F}_{b, a m p}, \text { and }-1 \leq \mathrm{W} \leq 1
$$

where $\mathrm{F}_{b}$ is the average of all interface elements and $\mathrm{F}_{b, a m p}$ is the width of spread around $\mathrm{F}_{b}$ and $\mathrm{W}$ is taken randomly from a uniform distribution on $[-1,1]$. The disorder parameter $\varphi$ is defined as:

$$
\varphi_{i}=\mathrm{F}_{b, a m p} / \mathrm{F}_{b}
$$

The simulation is load-controlled and the nodal displacements are calculated using a standard solution approach for linear FEM. A preconditioned conjugate gradients method is used to solve the system. As preconditioner the incomplete Cholesky factorization (ICF) of the stiffness matrix $\underline{\mathrm{K}}$ is used. In cases where the elements do not experience large strains or rotations, the stiffness matrix $\underline{\mathrm{K}}$ remains almost the same. $\underline{\mathrm{K}}$ is merely updated to incorporate the element removal. Likewise the ICF is not recalculated every increment but only when calculation time for the ICF is expected to be shorter than the convergence time. The external force is changed in such a way as to just break exactly one element in each increment. When the interface elements are loaded with large forces perpendicular to their axes, they show a non-linear relation between their extension and the applied force. This second order effect is taken into account when determining which interface spring breaks.

\section{Results}

It has been established earlier in [9] that crack fronts in the first two types of samples (PETG layers confined between a glass and a metal surface) mentioned above move inhomogeneously in time and in space. This was true for fronts moving along the smooth PETG-glass interfaces as well as for cracks running along the rough PETG-metal interfaces. The findings are summarized in Fig. 2 that appears to show two regimes of propagation. 1. A regime in which disorder appears to dominate, and in which cracks propagate mainly by initiation of bursts that subsequently spread along a small part of the front. 2 . A regime in which the lateral movement associated with an instability may span the entire sample width. In this case the stress concentration associated with a forward burst dominates the propagation. Invoking the image of a stress aided thermally activated process the local stress concentration contributes 

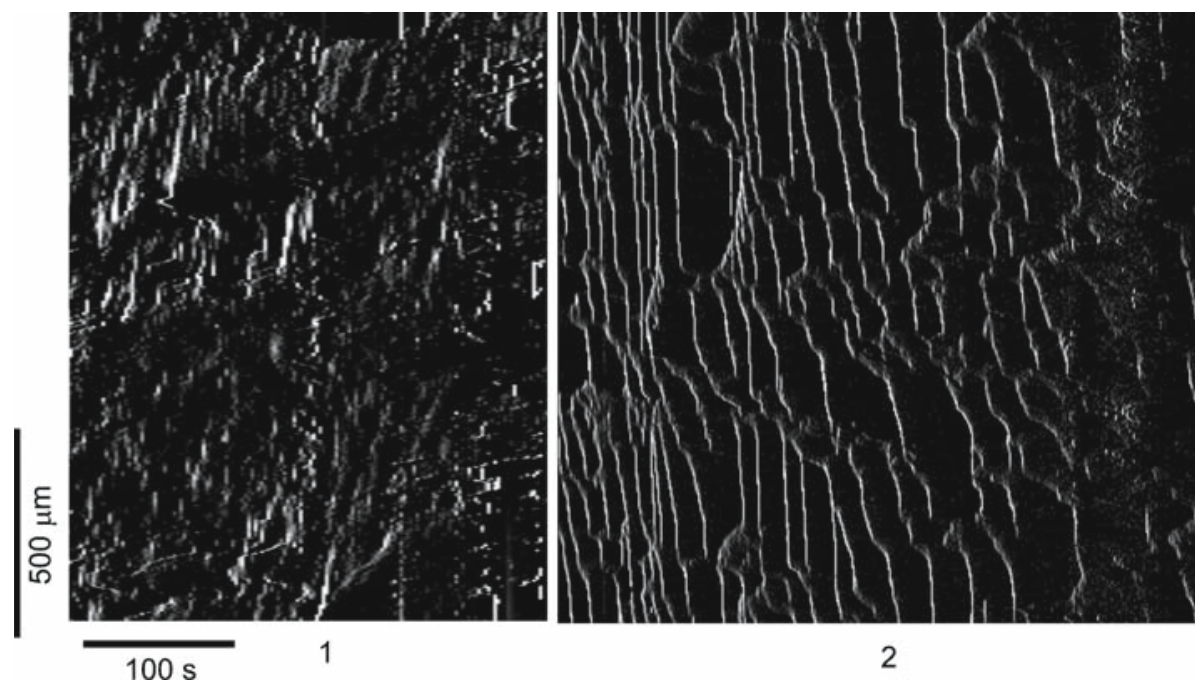

Fig. 2 Acceleration of crack front as a function of time (horizontal) and position along front (vertical). Light: high acceleration (forward burst), dark: low acceleration. Left: position of bursts mainly determined by disorder Right: position of bursts mainly determined by stress concentration near already existing protrusions in front

to a decrease in the energy barrier associated with delamination which leads locally to an increased probability of delamination.

Qualitatively similar crack front propagation phenomena have been reported in literature. Similar to regime 1 for polymer-polymer (PS-PS) interfaces [7, 8], and similar to regime 2 for epoxy-glass interfaces [14]. This seems to indicate that in fact such a crack front movement is a quite common mechanism.

A correlation between crack front propagation with disorder in the confined layer - in this case the interface roughness or related thickness differences - was encountered, and was more obvious in a crack propagating along a rough surface see Fig. 3.

Figure 4 shows the results of simulations in which the effect of disorder on crack propagation was investigated. It turns out that for low disorder a burst once initiated will only propagate by moving laterally along the front, dominated by the stress concentration at the edges of the burst. For high disorder burst may also move further ahead of the front if weak spots occur there.

In the cases discussed so far the interfaces were rough and anisotropic because of the rolling treatment that had been given to the metal substrates. To reduce the effects of local thickness differences as much as possible the $\mathrm{Cr}$ coated glass substrates described above were used. The crack position was measured as a function of time after an initial crack length below the equilibrium crack length was set pushing the razor blade towards the (pre-)crack front. Subsequently the crack front started to move away from the razor at ever decreasing speeds. During these experiments the following was observed.

1. The microscopic crack front geometry depended on the crack speed; there was a transition from essentially smooth crack fronts to rough, serrated crack fronts as the crack speed decreased (Fig. 5).

2. The macroscopic geometry of the crack front depended on the position with respect to the central axis of the sample, and was more or less symmetric with respect to the centre of the crack. This is believed to be associated with residual stresses in the coating (Fig. 6). 

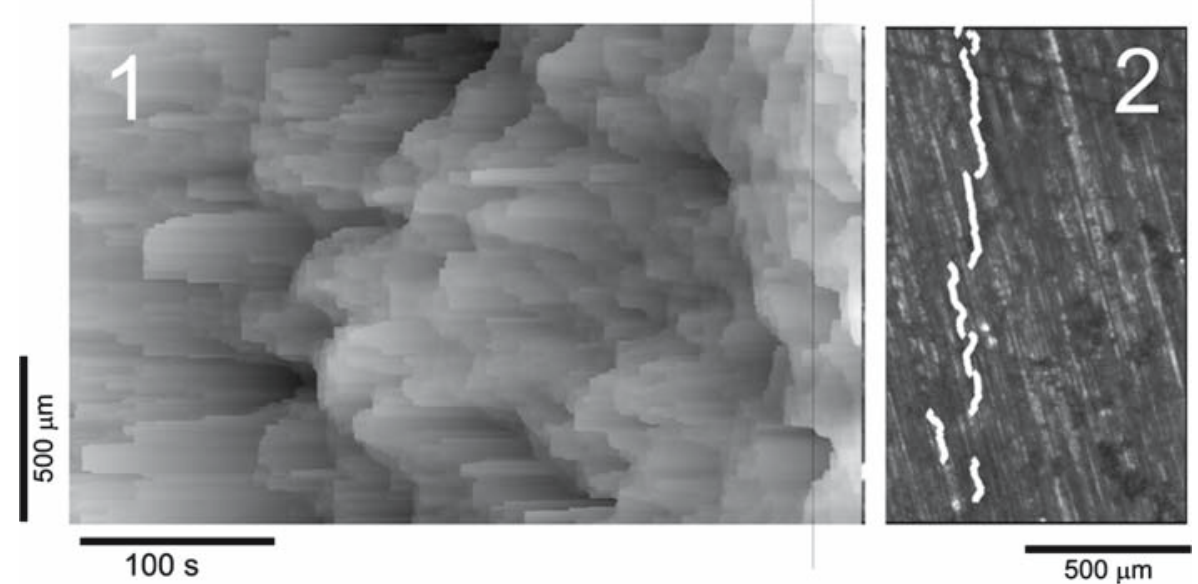

Fig. 3 Crack propagating along a rough anisotropic PETG-Al interface. 1: $\Delta x_{i}(t)=x_{i}(t)-\bar{x}_{i}(t)$, with $x$ position of front. 2 snapshot of front at position of the black line indicated in $\mathbf{1}$. The front position was determined as outlined in [9] and superimposed on a grayscale version of the original image. Note the alignment of parts of the front with the ridges on the metal surface
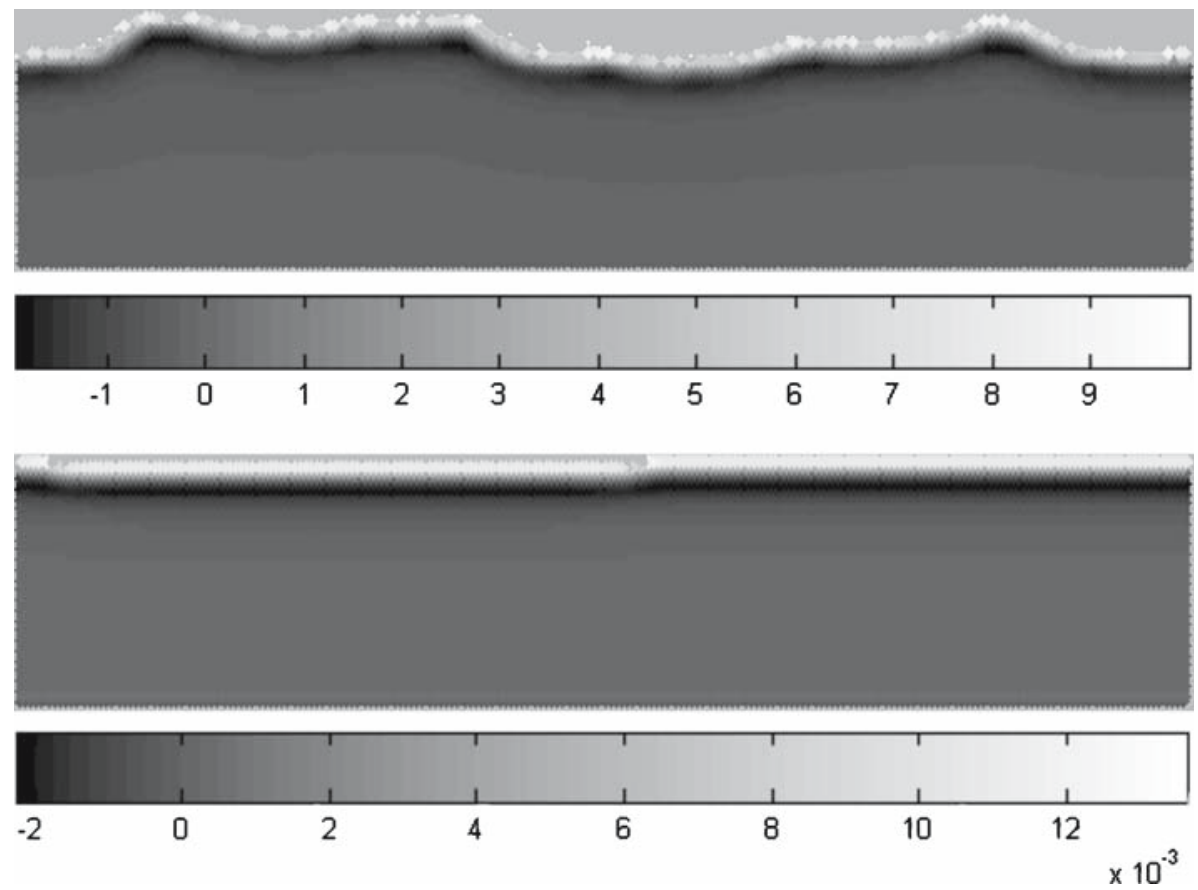

Fig. 4 Crack propagation in a lattice model (200*51 interface springs). In both cases the crack moves downwards. Dots indicate single interface springs, of which $200 * 51$ were initially present. Gray levels indicate forces in interface springs in the model. White corresponds to high tensile forces, black to compressive forces. Note areas of compression behind the crack front. Bottom: low disorder $\varphi=0.01$. Propagation is determined by stress concentrations only. Forward bursts along of the front grow in size by propagating along the crack front. Top: high disorder $\varphi=0.5$. Propagation may occur near stress concentrations as well as at inherently weak spots 


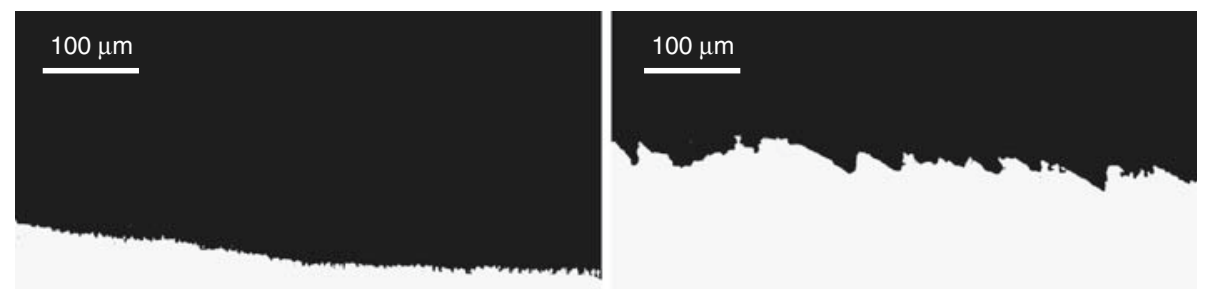

Fig. 5 Front geometry at different front speeds, from optical micrographs. Crack moves from bottom to top along a Cr-PETG interface. Left: Front geometry at higher speeds (order of $1 \mu \mathrm{m} / \mathrm{s}$ to $0.01 \mu \mathrm{m} / \mathrm{s}$ ). Right: Rough, serrated front geometry at very low speeds $(<1 \mathrm{~nm} / \mathrm{s})$

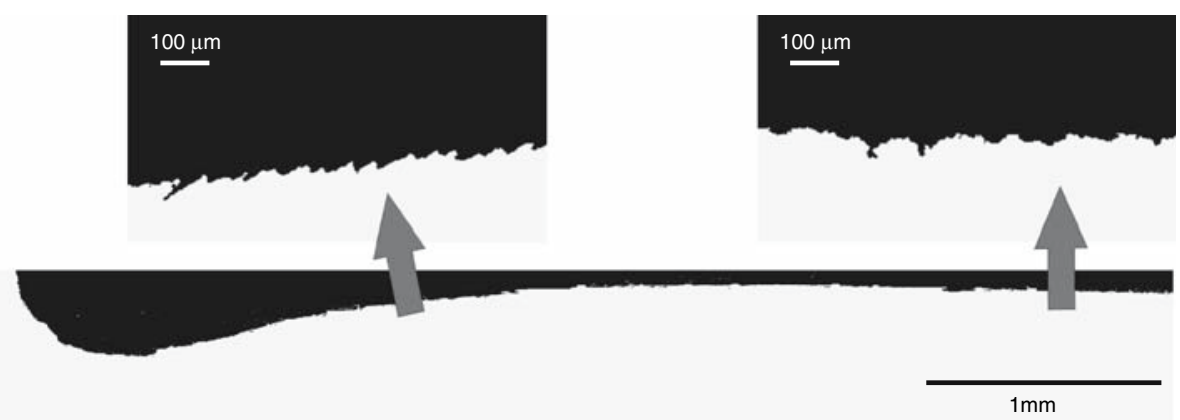

Fig. 6 Macroscopic (bottom, front shown from edge to centre-line of sample) and microscopic crack front geometry top at very low front speeds $(<1 \mathrm{~nm} / \mathrm{s})$ derived from optical micrographs. Crack moves from the bottom upwards, delaminated areas are white. Residual stress state likely causes macroscopic crack shape (compare Fig. 7). Different microscopic crack front geometry exists at different positions along the crack front. Top left: microscopic front geometry left of centre at position indicated by arrow. Top right: microscopic front geometry along the centre line

$\operatorname{Re} 1$. The change in crack front geometry can be understood invoking the physical picture of crack growth as a stress aided thermally activated process (subcritical crack growth) along an interface. Adopting for simplicity an Arrhenius description of the average dynamics of the crack movement (other formulations are possible, see [1]) and therefore disregarding the distinction between initiation of bursts at the crack front and propagation of already existing bursts, one can write:

$$
\mathrm{d} a / \mathrm{d} t=\mathrm{v}_{0} \operatorname{Exp}\left[-b\left(\mathrm{G}_{0}-\mathrm{G}(a, t)\right) / k T\right]
$$

where $a$ is the average crack-length, $\mathrm{G}(a)$ the associated energy release rate, $k T$ the thermal energy, and $b$ an area characteristic for the activated process. $\mathrm{G}_{0}$ is the energy barrier for delamination in the absence of a crack. Allowing for disorder $\mathrm{dG}_{0}(\boldsymbol{x})$ at position $\boldsymbol{x}$ passed by the front this becomes:

$$
\mathrm{d} a / \mathrm{d} t=\mathrm{v}_{0} \operatorname{Exp}\left[-b\left(\mathrm{G}_{0} \pm \mathrm{dG}_{0}(\boldsymbol{x})-\mathrm{G}(a, t)\right) / k T\right] .
$$




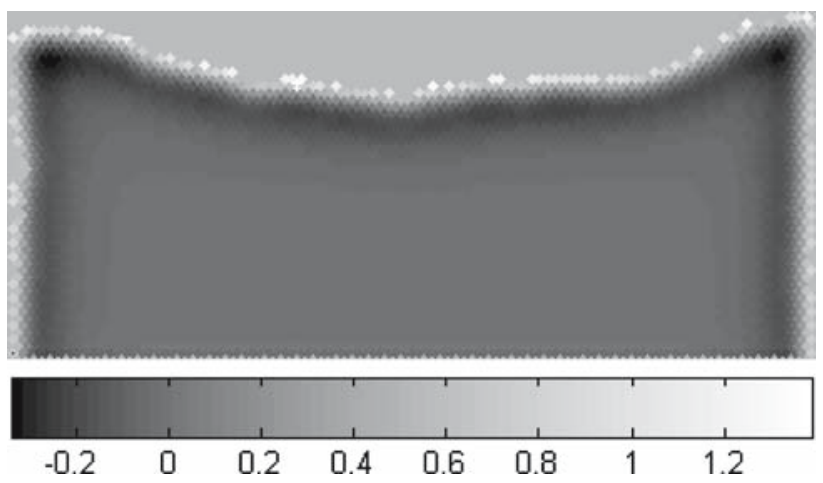

Fig. 7 Simulation $(100 * 51$ interface springs, $\varphi=0.5)$ of delamination along an interface with biaxial tensile stress condition superimposed, mimicking residual (thermal) tensile stresses in coating. Crack propagates from the top down. Note the regions on the interface plane under compression, caused by the bending of the coating along the sides of the sample and the crackfront. Compressive forces on the springs are highest near the corners where they "pin" the crack front. Note similarity in shape with the macroscopic experimental result shown in Fig. 6

For low values of $a, \mathrm{G}(a)$ is large and $\mathrm{G}_{0}-\mathrm{G}(a)$ tends to be small. This means that the thermal barrier for propagation is reduced and can be surmounted frequently and independently at many places along the front by thermal activation. In this case a small enough $\mathrm{dG}_{0}(\boldsymbol{x})$ will not change $\mathrm{d} a / \mathrm{dt}$ appreciably and crack propagation is homogeneous along the front. After the crack has propagated a situation is reached where the contribution of $\mathrm{G}(a)$ is so small that propagation is seen to become pinned at certain positions $\boldsymbol{x}$ along the front. Presumably the local energy barrier for delamination $\mathrm{G}_{0}+\mathrm{dG}_{0}(\boldsymbol{x})$ is highest at such positions, some of which were associated with visible inhomogeneities at the crack face. Near these sites propagation continues, leading to a roughening of the front. In some cases stress concentration near the pinned points is enough to break the front loose and the front temporarily straightens out again until another pinning point becomes active.

Re 2. On the scale of the sample the crack front is curved, see Fig. 6, which must be related to the presence of residual thermal stresses in the coating. Considering the fact that $\mathrm{T}=150^{\circ} \mathrm{C}$ during application of the coating and an estimated difference of $4 \cdot 10^{-5}(1 / \mathrm{K})$ between the thermal expansion coefficients of PETG and glass, a thermal stress of the order of up to $20 \mathrm{MPa}$ can be expected (the exact value depending on the stress relaxation above $\mathrm{Tg}=75^{\circ} \mathrm{C}$ during the cooling), which is very appreciable, i.e. about half the yield stress of PETG. In the centre of the sample the stress at the interface is expected to be bi-axial. Near the edges of the sample the situation is more complex as the normal stress components vanish near the edge leading to high residual shear stresses at the interface near the sides. These decrease slowly away from the edges. The peak in shear stress may induce delamination on weak spots at the sides of the samples. Interestingly, bending of the coating will lead to a region of compressive stress near the edge (see Fig. 7, and e.g. [15]). Near the corners bending from two side of the sample together leads to even higher compressive stresses. This distribution of normal stress on the interface plane is thought to influence crack propagation on a macroscopic (sample) scale, compare for example the experimental result in Fig. 6 with the simulation in Fig. 7.

It is also interesting to note that the microscopic geometry of the crack front may depend on the position along the front, and therefore on the local stress state. As an example Fig. 6 shows the occurrence of a serrated sawtooth-like morphology toward the edges of the sample, contrasting with the morphology in the centre. 


\section{Conclusions}

Several aspects of crack propagation along polymer-metal and polymer-glass interfaces and of the crack front geometry at such interfaces have been examined. The findings illustrate the influence of disorder, of stress redistribution along the front, and of crack speed. Interpreting the movement of the crack as subcritical crack growth that is determined by a stress aided thermally activated delamination process these effects can be qualitatively understood. Stress concentrations along the front locally decrease the energy barrier for propagation and may dominate crack propagation, masking the effects of disorder. Differences in crack front geometry as a function of speed may occur. The residual stress state along a crack front has been shown to have an impact on crack front geometry on the scale of the sample as well as on a microscopic scale.

Acknowledgements This research was carried out under project number MC05223 in the framework of the Strategic Research programme of the Netherlands Institute for Metals Research in the Netherlands.

Open Access This article is distributed under the terms of the Creative Commons Attribution Noncommercial License which permits any noncommercial use, distribution, and reproduction in any medium, provided the original author(s) and source are credited.

\section{References}

1. Kook, S.-Y., Dauskardt, R.H.: Moisture-assisted subcritical debonding of a polymer/metal interface. J. Appl. Phys. 91(3), 1293-1303 (2002)

2. Wouters, O., Vellinga, W.P., van Tijum, R., De Hosson, J.Th.M.: On the evolution of surface roughness during deformation of polycrystalline aluminum alloys. Acta Mater. 53(15), 4043-4050 (2005)

3. van Tijum, R., Vellinga, W.P., De Hosson, J.Th. M.: Acta Mat 55(8), 2757 (2007).

4. Fedorov, A., van Tijum, R., Vellinga, W.P., De Hosson, J.Th. M.: J. App. Phys. 101(4), 043520 (2007).

5. Sha, Y., Hui, C.Y., Kramer, E.J., Hahn, S.F., Berglund, C.A.: Fracture toughness and failure mechanisms of epoxy/rubber-modified polystyrene(PS) interfaces reinforced by grafted chains. Macromolecules 29, 4728-4736 (1996)

6. Bouchaud, E.: The morphology of fracture surfaces: a tool to understand crack propagation in complex materials. Surf. Rev. Lett. 10, 797 (2003)

7. Schmittbuhl, J., Måløy, K.J.: Direct observation of self-affine crack propagation. Phys. Rev. Lett. 78, 3888-3891 (1997)

8. Måløy, K.J., Schmittbuhl, J., Hansen, A., Batrouni, G.G.: Scaling and dynamics of an interfacial crack front. Int. J. Fract. 121, 9 (2003)

9. Vellinga, W.P., Timmerman, R., van Tijum, R., De Hosson, J.Th.M.: In situ observations of crack propagation mechanisms along interfaces between confined polymer layers and glass. Appl. Phys. Lett. 88, 06912 (2006)

10. Santucci, S., Vanel, L., Ciliberto, S.: Subcritical statistics in rupture of fibrous materials: experiments and model. Phys. Rev. Lett. 93, 095505 (2004)

11. Hutchinson, J.W., Suo, Z.: Mixed mode cracking in layered materials. Adv. Appl. Mech. 29, 63199 (1992)

12. Bernard, B., Brown, H.R., Hawker, C.J., Kellock, A.J., Russell, T.P.: Adhesion of polymer interfaces reinforced with random and Diblock copolymers as a function of geometry. Macromolecules 32, 62546260 (1999)

13. van den Bosch, M., Vellinga, W.P., Geers, M.G.D.: J. Fracture. (submitted)

14. Swadener, J.G., Liechti, K.M., de Lozanne, A.L.: The intrinsic toughness and adhesion mechanism of a glass/epoxy interface. J. Mech. Phys. Solids 47, 223-258 (1999)

15. He, M.Y., Evans, A.G.: Criterion for the avoidance of edge cracking in layered systems. J. Am. Cer. Soc. 87(8), 1418-1423 (2004) 